\title{
O Método Analytic Network Process na Instalação de Polos de Ensino a Distância: Uma Análise Bibliométrica
}

\section{The Method Analytic Network Process in the Installation of Distance Learning Poles: A Bibliometric Analysis.}

\author{
Fabrício Martins Carvalho da Silva1*, Júlio César Andrade de Abreu² \\ 1 Universidade Federal Fluminense \\ ¿Universidade Federal da Bahia
}

*Autor para correspondência: fabriciocarvalho@id.uff.br

\begin{abstract}
RESUMO
Esta pesquisa tem como objetivo mapear a produção científica em apoio multicritério no método Analytic Network Process aplicado à instalação de polos de ensino a distância, identificando os periódicos, autores, países e as instituições que mais publicaram sobre o tema, e dessa forma propor a futuros pesquisadores um método para desenvolver um "núcleo de partida" de uma pesquisa bibliográfica. Foi utilizado um modelo proposto de webibliomining, na base de dados Web of Science, que analisou a produção científica no período de 2003 a 2017. Os procedimentos metodológicos se utilizaram de uma pesquisa descritiva e exploratória, com abordagem quantitativa. Os resultados apontaram 11.221 artigos, e após uma análise bibliométrica constatou-se que o "pico" da produção foi no ano de 2017 com 1.556 artigos. Os Estados Unidos foram responsáveis por 25,30\% do total de artigos publicados, sendo a Educação a área que mais publicou artigos, 24,97\%. Foram selecionados trinta artigos para compor o "núcleo de partida" para uma futura pesquisa bibliográfica.
\end{abstract}

Palavras-Chave: Produção Científica; Webibliomining; Ensino a Distância; Multicritério.

\begin{abstract}
This research aims to map the scientific production in multicriteria support in the Analytic Network Process method applied to the installation of poles of distance learning, identifying the periodicals, authors, countries and institutions that published the most on the subject, and thus propose to future researchers a method to develop a "starting nucleus" of a bibliographical research. A proposed model of webibliomining was used in the Web of Science database, which analyzed the scientific production from 2003 to 2017. The methodological procedures made use of a descriptive and exploratory research, with a quantitative approach. The results pointed out 11,221 articles, which after a bibliometric analysis showed that the "peak" of the production was in 2017 with 1,556 articles. The United States accounted for $25.30 \%$ of the total number of articles published, with Education being the area with most published articles, $24.97 \%$, and thirty articles were selected to compose the "starting nucleus" for future bibliographic research.
\end{abstract}

Keywords: Scientific Production; Webibliomining; Distance Education; Multicriteria.

\section{Introdução}

A Educação a Distância (EaD) é uma modalidade de educação que tem crescido no Brasil. Segundo Arruda e Arruda (2015, apud SILVA; HERNÁNDEZ \& ABREU, 2017), a EaD surge como uma política pública em educação para expandir o número de vagas no ensino superior, ampliando o número de alunos nas universidades, não exigindo grandes estruturas físicas para o processo de ensino e aprendizagem, visto que parte de tal processo é realizada pelo aluno em tempo e espaço definidos por ele (SILVA; HERNÁNDEZ \& ABREU, 2017).

De acordo com dados do Censo da Educação Superior de 2017, divulgados pelo Instituto Nacional de Estudos e Pesquisas Educacionais 
Anísio Teixeira (Inep), as matrículas nos cursos de educação a distância no ano de 2017, 1.756.982, cresceram 17,57\% em relação ao ano de 2016, 1.494.418, enquanto que, nos cursos presenciais, houve uma queda de $0,38 \%$ no número de matrículas em 2017, 6.529.681, em relação a 2016, 6.554 .283 .

Segundo Belloni (2008), a Educação a Distância é uma modalidade de educação que aparece como uma solução necessária para atender às demandas sociais, permitindo a flexibilização do acesso da população ao ensino.

Segundo a legislação brasileira, a EaD deve ser semipresencial, isto é, exige que existam momentos presenciais obrigatórios destinados à realização de avaliações, aulas em laboratórios e tutorias (BRASIL, 2007). Esses momentos presenciais são cumpridos nos polos de apoio presencial.

O polo de apoio presencial é uma extensão da universidade, que cria condições de permanência do aluno no curso e possibilita o desenvolvimento regional e de geração de empregos (MOTA, 2009). É o local onde o aluno encontra lugar para estudar, interagir com pessoas, realizar avaliações e práticas laboratoriais (KNUPPEL, 2017).

A aplicação de técnicas multicritério auxilia o gestor na tomada de decisão, o que pode permitir a escolha do município mais apto à instalação de um polo de apoio presencial. Sendo assim, mapear a produção científica é o início para conhecer estudos realizados nesta área.

A técnica multicritério de apoio à decisão escolhida para esta pesquisa é o Analytic Network Process (ANP): ele analisa um problema de decisão organizando-o em uma estrutura em rede, possibilitando relações de dependência e feedback entre seus elementos (SAATY, 2005).

Conforme Saaty (2013, apud SILVA \& ABREU, 2019), no método ANP são feitos julgamentos pelo tomador de decisão e obtêm-se os vetores de prioridade que são combinados em toda a estrutura para dar o melhor resultado a uma decisão. Caso os julgamentos sejam inconsistentes, podem ser revisados pelo tomador de decisões, de maneira a tornar aceitável uma decisão, ou ela pode ser adiada até se obterem informações mais consistentes.

O interesse pela utilização do método ANP, dentre outras técnicas multicritério de apoio à decisão, reside no fato de este método avaliar se a estrutura de decisão possui dependência, ou seja, se existem fatores que interagem, obtendo maior precisão nos resultados (SAATY, 2005).

Uma pesquisa nas bases de dados Scielo e Google Acadêmico demonstrou a existência de estudos que tratam da utilização de técnicas bibliométricas para analisar artigos na área de auxílio multicritério à decisão.

Pereira e Costa (2015) realizaram um estudo bibliométrico em artigos indexados na base de dados Web of Science, referente a métodos multicritério de apoio à decisão: analisaram aspectos relacionados a autores, países de origem, quantitativos por ano, áreas de conhecimento, periódicos proeminentes e instituições de artigos identificados.

Peixoto Filho (2016) procedeu a um estudo bibliométrico, com o modelo de Webibliomining proposto por Costa (2010), em artigos na base Scopus: apresentou uma análise voltada para a produção científica na área de auxílio multicritério à decisão aplicada ao ensino a distância. Porém, não foram identificados estudos de webibliomining em artigos na base Web of Science referentes à aplicação do método de apoio multicritério ANP para instalação de polos de ensino a distância, nem com a quantidade de artigos selecionados para compor o "núcleo de partida" da pesquisa bibliográfica.

O objetivo desta pesquisa é identificar os periódicos, autores, países e as instituições que mais publicaram sobre o tema da pesquisa, mediante um modelo de webibliomining para garimpagem de textos na web. Dessa forma, pretende-se desenvolver um "núcleo de partida" para uma pesquisa bibliográfica que busca construir um modelo, baseado no método ANP, a fim de escolher municípios para a instalação de polos de apoio presencial para cursos de ensino superior a distância de universidades públicas; esse modelo poderá auxiliar na tomada de decisão dos gestores, selecionando os municípios que apresentam as melhores condições 
para sediar um polo permanente e duradouro, evitando prejuízos ao Erário.

A partir dessa introdução, o estudo foi elaborado da seguinte forma: a segunda seção apresenta uma revisão da literatura acerca da bibliometria; a terceira seção descreve os procedimentos metodológicos; a quarta traz a aplicação do modelo de webibliomining e os resultados; na quinta, é apresentada a conclusão.

\section{Da Bibliometria à Webibliomining: bre- ves considerações}

O termo Bibliometria (Bibliometrics) surgiu pela primeira vez em 1969, em um artigo de Alan Pritchard intitulado "Statistical Bibliography or Bibliometrics?". Pritchard (1969) definiu a Bibliometria como a aplicação de matemática e métodos estatísticos para livros e outros meios de comunicação.

Segundo Alvarado (1984), a bibliometria comporta três leis básicas: a lei de Bradford, que descreve a distribuição da literatura periódica em uma área específica; a lei de Lotka, que mostra a produtividade dos autores, e a lei de Zipf, que descreve a frequência de uso de palavras num determinado texto. Para Amaral et al. (2004), em linhas gerais, a bibliometria busca descobrir a variação de determinados elementos em um elemento fixo, isto é, um estudo mais quantitativo das publicações, tais como: os autores que mais publicam em um periódico, as palavras-chave que descrevem determinado tema, os autores mais citados por um autor em particular, dentre outras possibilidades.

A partir do surgimento da bibliometria, foram aparecendo novas áreas responsáveis pela medição da informação voltadas à internet e aos bancos de dados eletrônicos, tais como: Informetrics (Informetria), Webmetrics (Webometria), Bibliomining (Bibliomineração) e, mais recente, a Webibliomining (Webibliometria). Wormell (1998) definiu a informetria como um subcampo emergente da ciência da informação, que se baseia na combinação de técnicas avançadas de recuperação da informação com estudos quantitativos dos fluxos da informação.Já a Webometria, segundo Almind e Ingwersen (1997), citados por Vanti (2002), é uma área que surgiu a partir da informetria, que consiste na aplicação de métodos informétricos na WorldWide Web. O termo Bibliomining (Bibliomineração) refere-se à mineração de dados aplicada à biblioteca, através da utilização de ferramentas estatísticas, ou até mesmo de softwares, e do reconhecimento de padrões aplicados a dados produzidos em sistemas de bibliotecas (NICHOLSON, 2004).

Para Nicholson (2004), o processo da bibliomineração consiste em:

Determinar áreas temáticas de interesse; identificar as fontes de dados externas e internas; coletar, depurar e tornar anônimos os dados destinados a um repositório; selecionar ferramentas de análise apropriadas; descobrir padrões por meio da mineração de dados e pela produção de relatórios com ferramentas tradicionais de análise; analisar e implementar os resultados dessa atividade (NICHOLSON, 2004, p. 2).

Para Costa (2010), a Webibliomining é uma área de medição da informação que integra conceitos de Bibliometria, Webometria, Informetria e Bibliomineração, tendo por objetivo estabelecer um conjunto inicial de referências bibliográficas que permitirão o desenvolvimento de pesquisas em determinados temas, a partir de bases de dados disponíveis na rede web.

Segundo Gaspar, Shimoya e Shimoda (2018), a webibliomining consiste em:

Proporcionar uma investigação entre as referências, o que resulta em um estudo preliminar contendo trabalhos que farão parte do "núcleo de partida", garantindo ao pesquisador embasamento suficiente para o próprio estudo bibliográfico (GASPAR; SHIMOYA \& SHIMODA, 2018, p. 2).

Desta maneira, o fato de integrar conceitos de Bibliometria, Webometria, Informetria e Bibliomineração torna a webibliomining uma ferramenta mais eficaz para realizar estudos bibliográficos na web. 


\section{Metodologia}

A presente pesquisa caracteriza-se como descritiva e exploratória com abordagem quantitativa, por meio da análise bibliométrica de artigos dispostos em uma base de dados na rede web. Isso possibilitou coletar um conjunto de informações, permitindo identificar os textos mais relevantes para iniciar uma pesquisa acadêmica.

Os procedimentos metodológicos que foram definidos para o estudo bibliométrico são os do modelo de Webibliomining proposto por Costa (2010), que pode ser incluído na interseção entre a Bibliometria, Bibliomineração, Informetria e Webometria.

Esse modelo foi desenvolvido para fornecer a um pesquisador recém-ingresso em uma área de conhecimento a seleção de um núcleo inicial de artigos para sua pesquisa bibliográfica (COSTA, 2010), considerando a execução das seguintes etapas: (1) definição da amostra da pesquisa; (2) pesquisa na amostra, com as palavras-chave; (3) identificação dos periódicos com maior número de artigos publicados sobre o tema; (4) identificação dos autores com o maior número de publicações; (5) levantamento da cronologia da produção, identificando "ciclos de maior produção"; (6) seleção dos artigos para a composição do "núcleo de partida" para a pesquisa bibliográfica. Esse núcleo deve contemplar: (a) os artigos mais relevantes; (b) a identificação dos primeiros autores a escreverem sobre o tema; (c) a identificação dos últimos autores a escreverem sobre o tema; (d) a identificação dos textos mais relevantes em cada "ciclo de maior produção".

Contudo, para melhor acurácia nos resultados apresentados para esta pesquisa, foram realizadas algumas adaptações na metodologia criada por Costa (2010), sendo acrescentadas três etapas, a fim de identificar quais países apresentam maior interesse sobre o tema e mapear um volume maior de dados em diferentes periódicos e instituições. São elas: (1) a identificação dos países que mais publicaram artigos referentes ao tema; (2) a identificação das áreas de pesquisa que concentraram os maiores números de artigos; (3) a identificação das instituições mais representativas na área.

\section{Apresentação, Análise e Discussão dos Resultados da Aplicação do Modelo}

\subsection{Definição da Amostra}

Também foram definidas as quantidades de artigos que seriam selecionados para compor cada uma das etapas da pesquisa, conforme critérios estabelecidos pelos autores da pesquisa.

A amostra pesquisada corresponde aos artigos indexados na Base de Dados Web of Science, com acesso pelo Portal de Periódicos Capes, no mês de abril do ano de 2018.

A escolha por essa base reside no fato de a Web of Science possibilitar a identificação de artigos de periódicos em diversas áreas do conhecimento, com 150 milhões de registros, que incluem artigos, patentes, conjunto de dados, livros e mais. Sua base de dados é atualizada semanalmente, conta com a facilidade de acesso pelo portal de periódicos da Capes e ainda disponibiliza acesso a mais de 9.200 títulos de periódicos, podendo ser utilizada para uma pesquisa de alta qualidade, associando registros, buscando por autores relevantes na área de interesse, por referência citada, recebendo alertas de publicação para assuntos, publicações ou autores, processando os resultados de uma busca e salvando-os ou enviando-os por e-mail, salvando o histórico de buscas, entre outras possibilidades (BIBENG, 2014).

\subsection{Pesquisa na Amostra}

Coletaram-se dados em artigos publicados no período de 2003 a 2017, o que corresponde a um levantamento de quinze anos. O período utilizado equivale ao crescimento dos cursos superiores de Ensino a Distância no país, com o consequente aumento de publicações sobre o tema.

Primeiramente foi realizada uma busca na base de dados Web of Science, no campo de busca "Tópico", cuja amplitude corresponde aos títulos dos artigos, resumos, palavras-chave do autor, palavras-chave criadas (keywords plus). Essa busca foi feita aplicando-se o operador booleano OR, cuja funcionalidade permite encontrar pelo menos um dos termos digitados. Sendo assim, a chave de busca, adaptada de Peixoto Filho (2016), gerada 
para a seleção dos artigos, foi: Tópico: (Distance Education) OR Tópico: (Multicriteria Decision Model) OR Tópico: (Method ANP) OR Tópico: (Support Classroom Poles). Foram encontrados 17.912 registros, conforme a tabela 1 .

Numa segunda etapa, refinando-se os resultados encontrados na pesquisa para o tipo de documento "artigos", contabilizaram-se 11.221 ocorrências. Após essa etapa, foi iniciada a análise bibliométrica, cujos resultados serão apresentados nas seções seguintes.

\subsection{Identificação dos Periódicos com Maior} Número de Artigos Publicados sobre o Tema

Utilizando os recursos de busca da base Web of Science, foi possível identificar os dez periódicos com maior abertura a publicações no tema, conforme a tabela 2. O periódico com o maior número de artigos publicados é o Computers d Education, com 244 artigos.

\subsection{Identificação dos Autores com o Maior Número de Publicações}

Na tabela 3 foram considerados os autores que tivessem 18 ou mais publicações. G. H. Tzeng, da Universidade Nacional de Taipei, com 48 artigos, foi o autor com o maior número de publicações, inclusive na área Multicritério. A. T. de Almeida foi o autor com maior representatividade na área de ensino a distância, com 23 artigos publicados.

4.5. Levantamento da Cronologia da Produção, Identificando "Ciclos de Maior Produção"

No gráfico 1 é possível observar a evolução da produção de artigos no período de 2003 (345 artigos) a 2017 (1.556 artigos). Também se nota que o pico no "ciclo da produção" ocorreu entre os anos de 2015 e 2017, com a maior quantidade de artigos produzidos nos últimos quinze anos, totalizando 4.304 (1.341 em 2015; $1.407 \mathrm{em} \mathrm{2016;}$ e 1.556 em 2017), o que corresponde a $38,35 \%$ dos artigos publicados sobre o tema.

\subsection{Identificação dos Países que Mais Publi- caram Artigos Referentes ao Tema}

De acordo com a tabela 4, que mostra os dez países que mais publicaram artigos científicos sobre o tema, os Estados Unidos lideraram com 2.839 artigos. O Brasil aparece na quinta posição, com 608 artigos publicados.

\begin{tabular}{|c|c|}
\hline Tipos de Documentos & Registros \\
\hline Article & 11.221 \\
\hline Proceedings Paper & 6.031 \\
\hline Review & 361 \\
\hline Editorial Material & 121 \\
\hline Book Review & 97 \\
\hline Meeting Abstract & 59 \\
\hline Bibliography & 9 \\
\hline Letter & 5 \\
\hline Book Chapter & 3 \\
\hline News Item & 3 \\
\hline Correction & 1 \\
\hline Software Review & 1 \\
\hline
\end{tabular}

Tabela 1 - Tipos de documentos. Fonte: Elaborada pelos autores. 


\begin{tabular}{|l|r|}
\hline Título do periódico & Número de artigos \\
\hline Computers \& Education & 244 \\
\hline International Review of Research in Open and Distance Learning & 162 \\
\hline European Journal of Operational Research & 124 \\
\hline Distance Education & 117 \\
\hline Expert Systems with Applications & 112 \\
\hline PlosOne & 91 \\
\hline Mathematical Problems in Engineering & 62 \\
\hline American Journal of Pharmaceutical Education & 59 \\
\hline International Journal of Engineering Education & 54 \\
\hline News Item & 3 \\
\hline Correction & 1 \\
\hline Software Review & 1
\end{tabular}

Tabela 2 - Periódicos com maior número de artigos publicados. Fonte: Elaborada pelos autores.

\begin{tabular}{|c|c|}
\hline $\begin{array}{c}\text { Autores com maior número de } \\
\text { publicações }\end{array}$ & Registros \\
\hline Tzeng, G. H. & 48 \\
\hline Zavadskas, E. K. & 43 \\
\hline Richardson, J. T. E. & 34 \\
\hline Kahraman, C. & 24 \\
\hline De Almeida, A.T. & 23 \\
\hline Greco, S. & 23 \\
\hline Liu, Y. & 21 \\
\hline Slowinski, R. & 20 \\
\hline Zopounidis, C. & 20 \\
\hline Turskis, Z. & 18 \\
\hline Zhang, Y. & 18 \\
\hline
\end{tabular}

Tabela 3 - Autores com maior número de publicações. Fonte: Elaborada pelos autores.

4.7. Identificação das Áreas de Pesquisa que Concentraram os Maiores Números de Artigos

No gráfico 2 é possível observar as dez principais áreas de pesquisa do tema, assim como o número de artigos publicados. Na área de pesquisa Education Educational Research foram publicados 2.802 artigos, o que demonstra que a área da educação domina com publicações sobre o tema. 


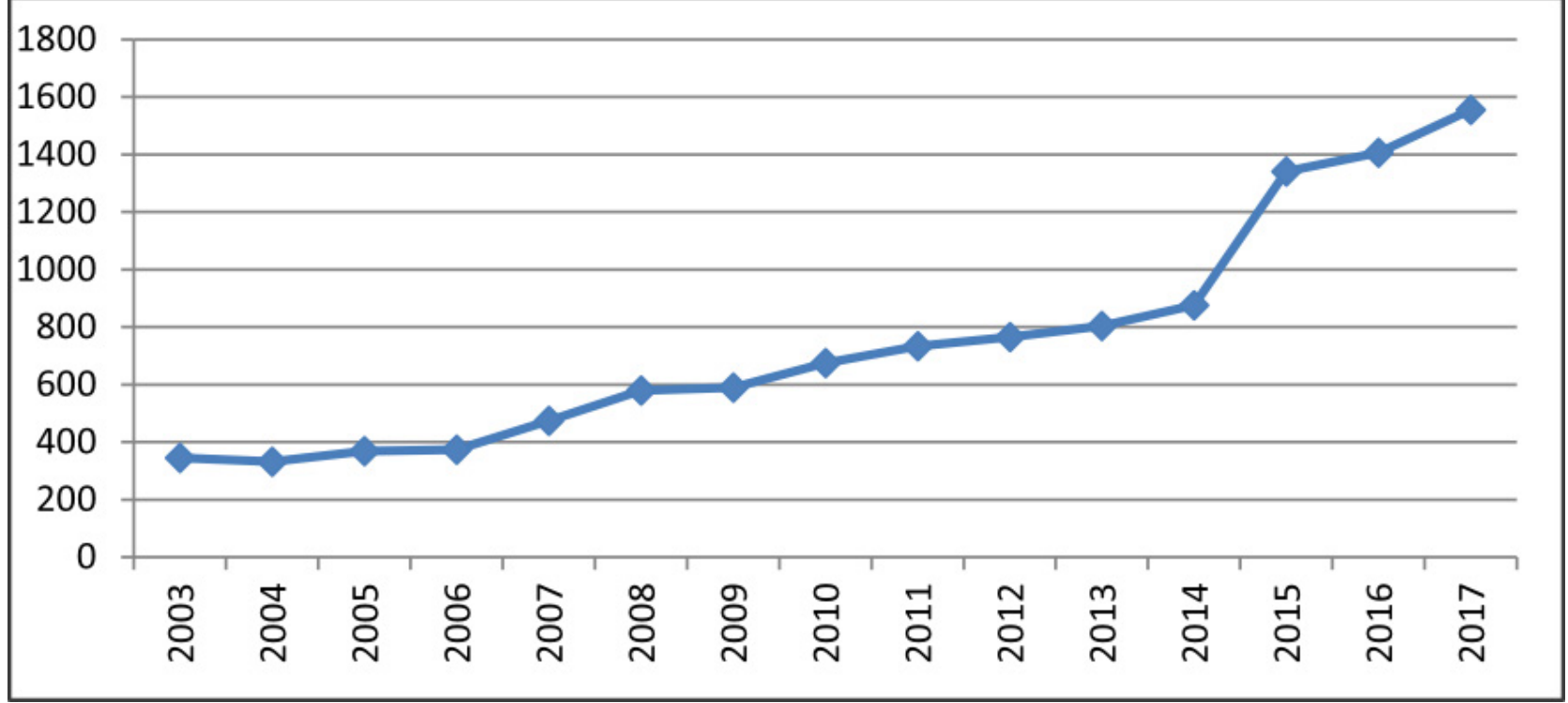

Gráfico 1 - Levantamento da cronologia da produção. Fonte: Elaborado pelos autores.

\begin{tabular}{|c|c|}
\hline Países & Artigos Publicados \\
\hline EUA & 2.839 \\
\hline China & 853 \\
\hline Inglaterra & 789 \\
\hline Espanha & 622 \\
\hline Brasil & 608 \\
\hline Canadá & 584 \\
\hline Austrália & 582 \\
\hline Taiwan & 491 \\
\hline Turquia & 486 \\
\hline Alemanha & 430 \\
\hline
\end{tabular}

Tabela 4 - Países que mais publicaram artigos. Fonte: Elaborada pelos autores.

4.8. Identificação das Instituições Mais Representativas na Área

No gráfico 3 , foram consideradas somente as instituições com 89 artigos ou mais. As universidades americanas lideraram esse ranking, com especial destaque para a University of California System, com 172 artigos publicados. A Universidade de São Paulo aparece na oitava posição, com 91 artigos publicados, o que demonstra a importância do ensino a distância no país.
4.9. Seleção dos Artigos para a Composição do "Núcleo de Partida" para a Pesquisa Bibliográfica

Para a realização desta etapa, foi definido o número de artigos que seriam selecionados a fim de compor o núcleo de partida para a pesquisa bibliográfica, disposto da seguinte forma: os dez artigos mais relevantes; a identificação dos quatro primeiros autores a escreverem sobre o tema; a identificação dos cinco últimos autores 


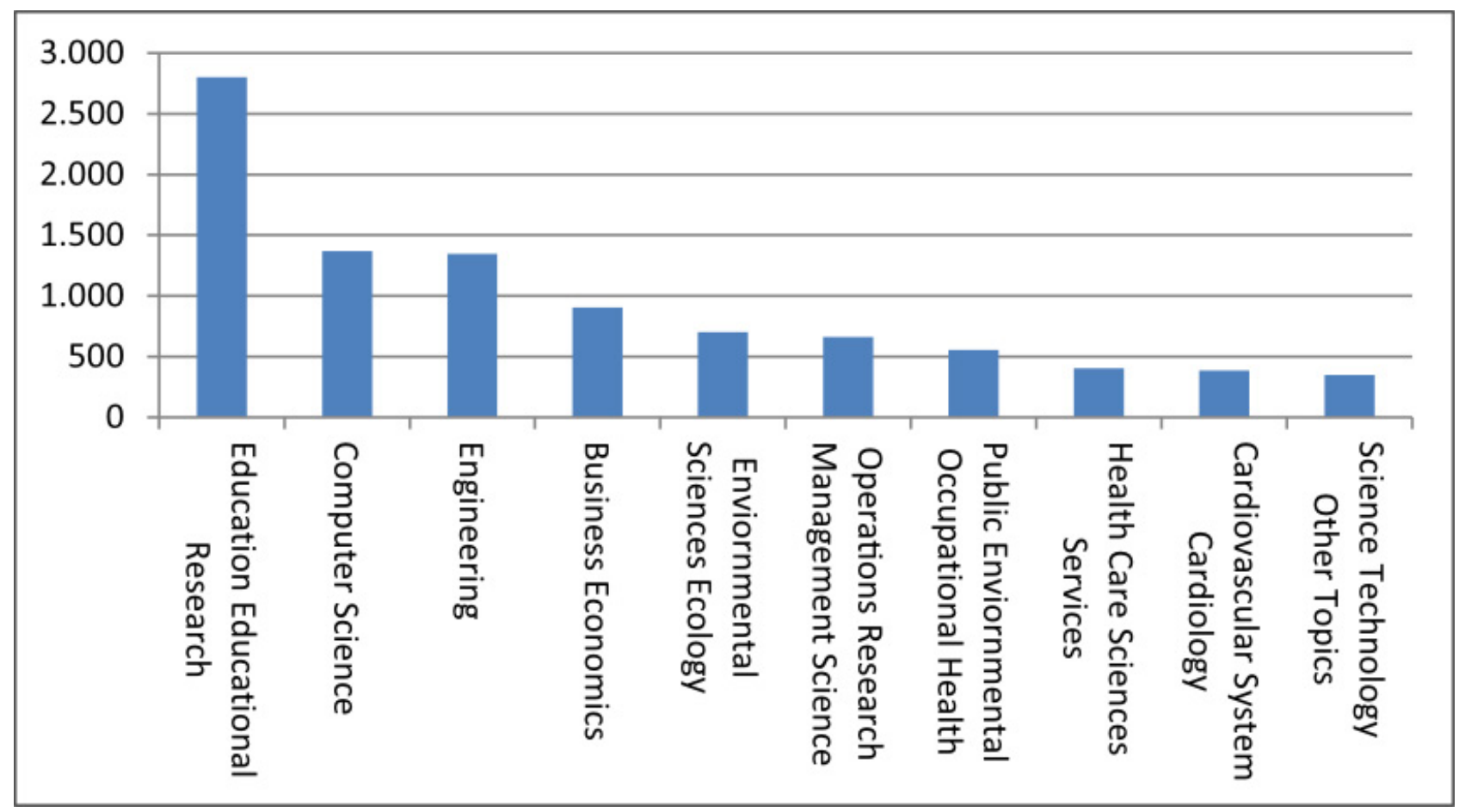

Gráfico 2 - Áreas de pesquisa que concentram os maiores números de artigos. Fonte: Elaborado pelos autores.

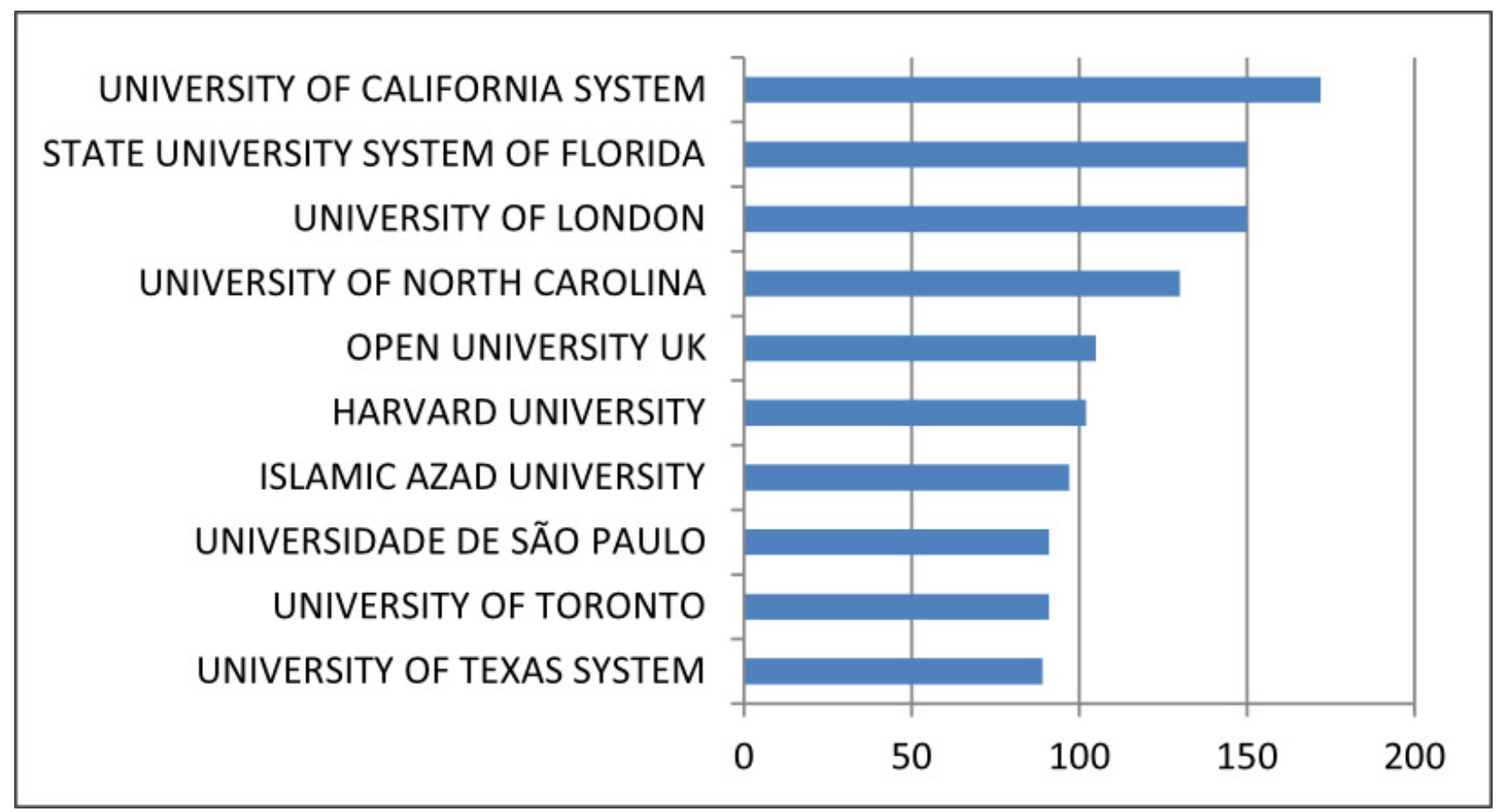

Gráfico 3 - Instituições e número de publicações. Fonte: Elaborado pelos autores.

a escreverem sobre o tema; a identificação dos cinco textos mais relevantes em cada "ciclo de maior produção"; e, adaptando a metodologia de Costa (2010), também foram inseridos seis artigos considerados interessantes para a pesquisa, totalizando trinta artigos para comporem o "núcleo de partida" para a pesquisa, conforme o quadro 1 . a) Os 10 artigos mais relevantes para a pesquisa foram: KABAK, M. et al. (2017); ARAGONES-BELTRAN, P. et al. (2010); SHANG, J. S.; TJADER, Y. \& DING, Y. (2004); KUMRU, M. \& KUMRU, P. Y. (2005); KAHRAMAN, C. et al. (2017); CHEN, Z. et al. (2014); VAHDANI, B.; HADIPOUR, H. 
\& TAVAKKOLI-MOGHADDAM, R. (2012); IVANOVIC, I. et al. (2013); KHADEMI, N.; MOHAYMANY, A. S. \& SHAHI, J. (2010); e NAKAGAWA, Y. et al. (2010).

b) Identificação dos 4 artigos mais antigos de autores diferentes presentes na base: VRASIDAS, G. (2003); LI, Q. H. (2003); DREW, S.; SHERIDAN, P. \& VENEMA, S. (2003); ABAS, Z. W. (2003).

c) Identificação dos 5 artigos mais recentes de autores diferentes presentes na base: $\mathrm{HONGOH}$, V. et al. (2017); ALEGRE DE LA ROSA, O. M. \& VILLAR ANGULO, L. M. (2017); TOBISZEWSKI, M.; NAMIESNIK, J. \& PENAPEREIRA, F. (2017); ASADABADI, M. R. (2017); CREA, T. M.; e SPARNON, N. (2017). d) Os 5 artigos mais relevantes no pico do "ciclo da produção", entre os anos de 2015 e 2017, conforme o gráfico 2, foram: BORGES SENA, P. M. \& CHAGAS, M. T. (2015); PATRAO AQUINO, G. N.; CALDAS PEREIRA, L. A. \& ERTHAL JUNIOR, M. (2017); CHANG, K. L. (2015); SCANAVACHI MOREIRA CAMPOS, A. C. \& DE ALMEIDA, A. T. (2016); e KOG, M. (2017).

e) Seleção dos 6 artigos considerados interessantes para a pesquisa, presentes na base: SAATY, T. L. (2013); GHENG, E. \& LI, H. (2005); SIPAHI, S. \& TIMOR, M. (2010); YAZGAN, H. R.; BORAN, S. \& GOZTEPE, K. (2010); GARCIA-MELON, M. et al. (2008) e SUN, P. G. et al. (2008).

ABAS, Z. W. "Incorporating Motivational Elements in a Web-Based Learning Environment for Distance Students: A Malaysian Experience". Lecture Notes in Computer Science, vol. 2783, pp. 396-410, 2003.

ALEGRE DE LA ROSA, O. M. \& VILLAR ANGULO, L. M. "Indicators and Statistical Control in Monitoring and Assessing University Students' Learning Preferences". Red-Revista de Educacion a Distancia, ed. 55, n. 2, 2017.

ARAGONES-BELTRAN, P. et al. "An Analytic Network Process Approach for Siting a Municipal Solid Waste Plant in the Metropolitan Area of Valencia (Spain)". Journal of Environmental Management, vol. 91, ed. 5, pp. 1071-1086, 2010.

ASADABADI, M. R. "A Customer Based Supplier Selection Process That Combines Quality Function Deployment, The Analytic Network Process And A Markov Chain". European Journal of Operational Research, vol. 263, ed. 3, pp. 1049-1062, 2017.

BORGES SENA, P. M. \& CHAGAS, M. T. "The University Library In Distance Education: Paper, Features And Challenges". Perspectivas em Ciência da Informação, vol. 20, ed. 4, pp. 163-180, 2015.

CHANG, K. L. "The Use of a Hybrid MCDM Model for Public Relations Personnel Selection". Informática, vol. 26, ed. 3, pp. 389-406, 2015.

CHEN, Z. et al. "ANP Experiment for Demolition Plan Evaluation". Journal of Construction Engineering and Management, vol. 140, ed. 2, n. 06013005, 2014.

CHENG, E. \& LI, H. "Analytic Network Process Applied to Project Selection". Journal of Construction Engineering and Management, vol. 131, ed. 4, pp. 459-466, 2005.

CREA, T. M. \& SPARNON, N. "Democratizing Education at the Margins: Faculty and Practitioner Perspectives on Delivering Online Tertiary Education for Refugees". International Journal of Educational Technology in Higher Education, vol. 14, n. 43, 2017.

DREW, S.; SHERIDAN, P. \& VENEMA, S. "Collaborative Supervision of Machine Learning as a Tool for Web-Based Education: A Teaching and Learning Triangle". Lecture Notes in Computer Science, vol. 2783, pp. 327-338, 2003.

GARCIA-MELON, M. et al. Farmland Appraisal Based on the Analytic Network Process". Journal of Global Optimization, vol. 42, ed. 2, pp. 143-155, 2008.

$\mathrm{HONGOH}, \mathrm{V}$. et al. "Criteria for the Prioritization of Public Health Interventions for ClimateSensitive Vector-Borne Diseases in Quebec". Plos One, vol. 12, ed. 12, n. e0190049, 2017. 
IVANOVIC, I. et al. "One approach for road transport project selection". Transport Policy, vol. 25, pp. 22-29, 2013.

KABAK, M. et al. "Evaluation of Distance Education Websites: a Hybrid Multicriteria Approach". Turkish Journal of Electrical Engineering and Computer Sciences, vol. 25, ed. 4, pp. 2809-2819, 2017.

KAHRAMAN, C. et al. "Intuitionistic Fuzzy Edas Method: an Application to Solid Waste Disposal Site Selection". Journal of Environmental Engineering and Landscape Management, vol. 25, ed. 1, pp. 1-12, 2017.

KHADEMI, N.; MOHAYMANY, A. S. \& SHAHI, J. "Intelligent Transportation System User Service Selection and Prioritization Hybrid Model of Disjunctive Satisfying Method and Analytic Network Process". Transportation Research Record, ed. 2189, pp. 45-55, 2010.

KOG, M. "Learning Analytics of Student Participation and Achievement in Online Distance Education: A Structural Equation Modeling". Educational Sciences-Theory o Practice, vol. 17, ed. 6, pp. 1893-1910, 2017.

KUMRU, M. \& KUMRU, P. Y. "A Fuzzy ANP Model for the Selection of 3D CoordinateMeasuring Machine". Journal of Intelligent Manufacturing, vol. 26, ed. 5, pp. 999-1010, 2015.

LI, Q. H. et al. "GridFS: A Web-Based Data Grid for the Distributed Sharing of Educational Resource Files". Lecture Notes in Computer Science, vol. 2783, pp. 81-92, 2003.

NAKAGAWA, Y. et al. "Analytic Hierarchy Based Policy Design Method (AHPo) for Solving Societal Problems that Require a Multifaceted Approach". European fournal of Operational Research, vol. 207, ed. 3, pp. 1545-1553, 2010.

PATRAO AQUINO, C. N.; CALDAS PEREIRA, L. A. \& ERTHAL JUNIOR, M. "Multicriteria Modeling to Establish Distance Education Centers in Mesoregions the Fluminense Federal Institute". Revista Tecnologia e Sociedade, vol. 13, ed. 28, pp. 90-110, 2017.

SAATY, T. L. "The Modern Science of Multicriteria Decision Making and Its Practical Applications: The AHP/ANP Approach". Operations Research, vol. 61, ed. 5, pp. 1101-1118, 2013.

SCANAVACHI MOREIRA CAMPOS; A. C. \& DE ALMEIDA, A. T. "Multicriteria Framework for Selecting a Process Modelling Language". Enterprise Information Systems, vol. 10, ed. 1, pp. 17-32, 2016.

SIPAHI, S. \& TIMOR, M. "The analytic hierarchy process and analytic network process: an overview of applications". Management Decision, vol. 48, ed. 5-6, pp. 775-808, 2010.

SHANG, J. S.; TJADER, Y. \& DING, Y. "A Unified Framework For Multicriteria Evaluation Of Transportation Projects". IEEE Transactions on Engineering Management, vol. 51, ed.3, pp. 300313, 2004.

SUN, P. C. et al. "What Drives a Successful E-Learning? An Empirical Investigation of the Critical Factors Influencing Learner Satisfaction". Computers \& Education, vol. 50, ed. 4, pp. 11831202, 2008.

TOBISZEWSKI, M.; NAMIESNIK, J. \& PENA-PEREIRA, F. "A Derivatisation Agent Selection Guide". Green Chemistry, vol. 19, ed. 24, pp. 5911-5922, 2017.

VAHDANI, B.; HADIPOUR, H.; TAVAKKOLI-MOGHADDAM, R. "Soft Computing Based On Interval Valued Fuzzy ANP-A Novel Methodology". Journal of Intelligent Manufacturing, vol. 23, ed. 5, pp.1529-1544, 2012.

VRASIDAS, C. "Concepts to Consider when Studying Computer-Mediated Communication and Online Learning". Lecture Notes in Computer Science, vol. 2563, pp. 232-247, 2003.

YAZGAN, H. R.; BORAN, S.; GOZTEPE, K. "Selection of Dispatching Rules in FMS: ANP Model Based on BOCR with Choquet Integral". International Journal of Advanced Manufacturing Technology, vol. 49, ed. 5-8, pp. 785-801, 2010.

Quadro 1 - Artigos do núcleo de partida para pesquisa. Fonte: Elaborado pelos autores. 


\section{Conclusões}

No estudo realizado, o ano de 2017 foi destaque no número de artigos sobre Educação a Distância, com um crescimento de $351 \%$ da produção científica em relação ao ano de 2003 , período inicial da pesquisa. Esse aumento no volume de artigos pode ser considerado como um reflexo do crescimento da modalidade de ensino a distância no período pesquisado.

O país que mais publicou artigos foram os Estados Unidos da América (EUA) com 2.839 artigos, o que corresponde a 25,30\% dos 11.221 artigos relacionados para esta pesquisa. Das dez instituições que mais publicaram artigos sobre os termos da pesquisa, cinco delas se localizam nos Estados Unidos: University of California System (172), State University System of Florida (150), University of North Carolina (130), Harvard University (102) e University of Texas System (89).

Conforme dados do site Moodle Livre (2018), os Estados Unidos, no ano de 2018, foram o líder mundial em educação on-line, com centenas de universidades a distância e milhares de cursos oferecidos nessa modalidade; no ano de 2011, 6 milhões de alunos estavam cursando ao menos um programa virtual, o que significa um em cada três estudantes matriculados no nível superior do país.

Segundo a pesquisa, o Brasil ficou na quinta posição, com 608 artigos publicados, na frente de países como: Canadá (584), Austrália (582) e Alemanha (430). A Universidade de São Paulo (USP) ficou na oitava posição, com 91 artigos publicados.

A produção brasileira na área de educação a distância reflete o baixo investimento do governo na área da educação, o que faz com que o ritmo das pesquisas se torne incompatível com o crescimento acelerado dessa modalidade de ensino. A valorização no país de cursos voltados às áreas de exatas e da saúde confirma o maior número de pesquisas nas áreas das engenharias e da medicina.

A área de pesquisa que teve mais artigos publicados foi Education Educational Research, com 2.802 artigos, o que equivale a $24,97 \%$ do total de artigos encontrados para esta pesquisa, seguida pela área Computer Science, com 12,20\% dos artigos pesquisados.

O interesse de publicar artigos nessas áreas se deve ao fato de a educação a distância ser uma modalidade de educação, logo as revistas científicas que se interessam pelo tema são da área educacional. Como o uso das tecnologias de informação e comunicação é essencial para o funcionamento dos cursos a distância e as tecnologias estão em constante inovação, as revistas científicas da área da Ciência da Computação têm maior interesse para publicar artigos sobre o tema tecnologias de informação e computação para cursos de $\mathrm{EaD}$ do que outras revistas científicas.

Dentre os artigos considerados interessantes para compor o núcleo de partida da pesquisa está o de Saaty (2013), um importante autor na área de multicritério, inventor do método Analytic Network Process (ANP), um dos termos de busca desta pesquisa e utilizado na construção do modelo para a seleção de municípios para a instalação de polos de apoio presencial, conforme o objetivo desta pesquisa. Logo, a leitura desse artigo se mostra fundamental para a realização do trabalho.

Dessa forma, a pesquisa realizada na base $W e b$ of Science atendeu aos objetivos propostos inicialmente, permitiu a criação de um núcleo de partida para a pesquisa, ampliando o conhecimento sobre a bibliografia da área, identificando os principais periódicos e autores da área e aumentando a qualidade da pesquisa.

Verificou-se como limitação o fato de a pesquisa ter sido feita em apenas uma base de dados, embora se entenda que a Web of Science é uma das maiores do mundo. Dessa maneira, sugere-se a realização do estudo em outras bases de dados, a fim de confirmar a eficácia do modelo proposto para uma pesquisa bibliográfica.

\section{Referências Bibliográficas}

ALVARADO, R. U. "A Bibliometria no Brasil". Ciência da Informação, vol. 13, n. 2, 1984, pp. 91-105.

AMARAL, R. M. et al. "Uma Visão da Produção científica nos Anais do Encontro 
Nacional de Engenharia de Produção através da Bibliometria". Encontro Nacional de Engenharia de Produção, 25, 2004, 03-05 nov.; Florianópolis-SC. Anais. Florianópolis-SC: Engenep, 2005.

ARRUDA, D. E. P. \& ARRUDA, E. P. "Educação a Distância no Brasil: Políticas Públicas e Democratização do Acesso ao Ensino Superior". Educação em Revista, Belo Horizonte, vol. 31, n. 3, jul./set. 2015, pp. 321-338.

BELLONI, M. L. Educação a Distância. São Paulo: Autores Associados, 2008.

BIBENG. Biblioteca da Escola de Engenharia da UFRGS. Web of Science - Tutorial, 2014. Disponível em: <https:/www.ufrgs.br/bibeng/ wp-content/uploads/2014/02/WEB_OF_ SCIENCE.pdP>. Acessado em 20 mar. 2018.

BRASIL. Ministério da Educação. Referenciais de Qualidade para a Educaşão Superior a Distância. Brasília, DF: Seed-MEC, 2007. Disponível em: $<$ http://portal.mec.gov.br/seed/arquivos/pdf/legislacao/refead1.pdf >. Acessado em 16 mar. 2019.

BRASIL. Ministério da Educação. Censo da Educação Superior, 2017. Disponível em: $<$ http:// portal.mec.gov.br/docman/setembro-2018-pdf/ 97041-apresentac-a-o-censo-superior-u-ltimo/file>. Acessado em 15 abr. 2019.

COSTA, H. G. "Modelo para Webibliomining: Proposta e Caso de Aplicação". Revista da FAE, vol. 13, n. 1, 2010, pp. 115-126.

GASPAR, I.A.; SHIMOYA, A. \&SHIMODA, E. "Extração de Informações de Laudos Médicos Utilizando Text Mining: Aplicação do Método Webibliomining". Acta Biomedica Brasiliensia, vol. 9, n. 2, 2018, pp.01-09.

KNUPPEL, M. A. C. "Polos de Educação a Distância no Brasil: Identidade e Representação". EmRede: Revista de Educaşão a Distância, vol. 4, n. 2, 2017, pp. 285-302.

MOODLE LIVRE.com. 5 Países que Lideram o Setor de Cursos a Distância no Mundo. Disponível em: <https://www.moodlelivre.com.br/noticias/ 3200-5-paises-que-lideram-o-setor-de-cursos-a-distancia-no-mundo>. Acessado em 25 maio 2019.

MOTA, R. "A Universidade Aberta do Brasil". In:LITTO, F. M. \& FORMIGA, M. M. M. Educasãa a Distância: o Estado da Arte. São Paulo: Pearson Education do Brasil, vol. 1, 2009, pp. 297-303.

NICHOLSON, S. "O Processo da Bibliomineração: Repositório de Dados e Mineração de Dados para Tomada de Decisão em Bibliotecas". Transinformação, vol. 16, n. 3, 2004, pp. 253-261.

PEIXOTO FILHO, J. Modelagem Multicriterial Aplicada a Seleção de Municípios para Abertura de Polos de Educação a Distância. 2016. 120 f. Dissertação (Mestrado em Engenharia de Produção), Universidade Cândido Mendes, Rio de Janeiro, 2016.

PEREIRA, F. G. \& GOSTA, H. G. "Métodos Multicritério de Apoio à Decisão: um Estudo Bibliométrico em Periódicos Indexados na Base Isi Web Of Science". Congresso Nacional de Excelência e Gestão, 11, 2015, 13-14 ago. Anais. Rio de Janeiro: Inovarse, 2015.

PRITCHARD, A. "Statistical Bibliography or Bibliometrics?". Journal of Documentation, vol. 25, n. 4, 1969, pp. 348-349.

SAATY, T. L. Theory and Applications of the Analytic Network Process: Decision Making with Benefits, Opportunities, Costs and Risks. Pittsburgh: RWS, 2005.

SAATY, T. L. "The Modern Science of Multicrit eria Decision Making and Its Practical Applications: The AHP/ANP Approach". Operations Research, vol. 61, ed. 5, 2013, pp. 1101-1118.

SILVA, F. M. C. \& ABREU, J. G. A. "Aplicação do Método de Análise em Rede na Instalação de Polos de Educação a Distância na Região Serrana Fluminense". Revista EDaPECI, Sergipe, vol. 19, n. 1, jan./abr. 2019, pp. 28-43

SILVA, F. M. G.; HERNÁNDEZ, G. T. \& ABREU, J. G. A. "Avaliação de Critérios para Instalação de Polos de Apoio Presencial para Cursos de Ensino a Distância Utilizando o Analytic Network Process". Competência, Porto Alegre, vol. 10, n. 2, dez. 2017.

VANTI, N. "Da Bibliometria à Webometria: uma Exploração Conceitual dos Mecanismos Utilizados para Medir o Registro da Informação e a Difusão do Conhecimento". Ciência da Informação, v.31, n.2, 2002, p.152-162. 
WORMELL, I. "Informetria: Explorando Ciência da Informação, Brasília, vol. 27, n. 2, 1998.

Bases de Dados como Instrumentos de Análise".

Publicado em 28/07/2020. 